\title{
On Load Balancing in a Dense Wireless Multihop Network
}

\author{
Esa Hyytiä and Jorma Virtamo \\ Networking Laboratory, Helsinki University of Technology, \\ P.O. Box 3000, FIN 02015 TKK, Finland
}

\begin{abstract}
We study the load balancing problem in a dense multihop network, where a typical path consists of large number of hops, i.e., the spatial scales of a typical distance between source and destination, and mean distance between the neighbouring nodes are strongly sepatated. In this limit, we present a general framework for analysing the traffic load resulting from a given set of paths and traffic demands. We formulate the load balancing problem as a minmax problem and give two lower bounds for the achievable minimal maximum traffic load. The framework is illustrated by an example of uniformly distributed traffic demands in a unit disk with a few families of paths given in advance. With these paths we are able to decrease the maximum traffic load by factor of $33-40 \%$ depending on the assumptions. The obtained traffic load level comes quite near the tightest lower bound.
\end{abstract}

Index Terms-multihop network, load balancing

\section{INTRODUCTION}

In a dense wireless multihop network a typical path consists of several hops and intermediate nodes along a path act as relays. In this paper we focus on studying the traffic load in such a network. By traffic load we mean, roughly speaking, the rate at which packets are transmitted in the proximity of a given node, and the objective of load balancing is to find such paths that minimise the maximum traffic load in the network. In particular, we assume a strong separation in spatial scales between the macroscopic level, corresponding to a distance between the source and destination nodes, and the microscopic level, corresponding to a typical distance between the neighbouring nodes. This assumption justifies modelling the routes on the macroscopic scale as smooth geometric curves as if the underlying network fabric formed a homogeneous, continuous medium.

The microscopic scale corresponds to a single node and its immediate neighbours. At this scale the above assumptions imply that only the direction in which a particular packet is traversing is significant. In particular, considering one direction at a time there exists a certain maximum flow of packets a given MAC protocol can support (packets per unit time per unit length, "density of progress"). Generally, this maximal sustainable directed packet flow depends on the particular MAC protocol defining the scheduling rules and possible coordination between the nodes. Determining the value of this maximum is not a topic of this paper but is assumed to be given (known characterstic constant of the medium). By a simple time sharing mechanism this maximal value can be shared between flows propagating in different directions. As a result, the scalar or total flux (to be defined in Section II) of packets is bounded by the given maximum, and the load balancing task is to determine the paths in such a way that the maximum flux in minimised.

Under the assumption of a dense multihop network the shortest paths (SP) are approximately straight line segments [PP03]. Straight paths yield an optimal solution in terms of mean delay when the traffic demands are low and there are no queueing delays. However, they typically concentrate significantly more traffic in the center of network than elsewhere, and as the traffic load increases the packets going through the center of the network start to experience queueing delays and eventually the system becomes unstable when the maximal sustainable scalar flux is exceeded. Hence, the use of shortest paths limits the capacity of the multihop network unnecessarily and our task is to minimise the maximum packet flux in the network by a proper choice of paths on the macroscopic scale.

Our main contribution is the formulation of the traffic load and the corresponding load balancing problem in a dense multihop network. For the load balancing problem we provide two lower bounds. Further, we show how the scalar flux can be calculated for a given set of curvilinear paths. Even though the results are valid only in the limit of a dense network (i.e., a large number of nodes and a small transmission range), they give insight and can serve as useful approximations for more realistic scenarios.

The rest of the paper is organised as follows. In Section II we present the necessary mathematical framework. In Section III two lower bounds for the achievable 
traffic load level are presented. In Section IV the general expressions for the traffic load with curvilinear paths are derived. In Section V we demonstrate the load balancing in unit disk with three different path sets yielding a better performance than the shortest paths in terms of maximum traffic load. Section VI contains our conclusions.

\section{A. Related Work}

In [PP03] Pham et al., and later in [GK04] Ganjali et al., have studied the load balancing using multipath routes instead of shortest paths. The analysis is done assuming a disk area and a high node density so that the shortest paths correspond to straight line segments. In multipath situation the straight line segments are replaced by rectangular areas where the width of the rectangle is related to the number of multiple paths between a given pair of nodes. In particular, multiple paths are fixed on both sides of the shortest path.

In [DBT05] Dousse et al. study the impact of interference on the connectivity of large ad hoc networks. They assume an infinite area and the behaviour of each node to be independent of the other nodes, which, together with interference assumptions, define the stochastic properties for the existance of links. With these assumptions the authors study the existance of a gigantic component, which is related to the network connectivity.

In [SMS05] Sirkeci-Mergen et al. study a dense wireless network with cooperative relaying, where several nodes transmit the same packet simultaneously in order to achieve a better signal-to-noise ratio. In the analysis an infinitely long strip is studied and the authors are able to identify a so-called critical decoding treshold for the decoder above which the message is practically transmitted to any distance (along the strip). The analysis assumes a dense network similarly as in this paper.

In a dense network with shortest path routing the transmission of each packet corresponds to a line segment in the area of the network. A line segment process with uniformly distributed end points is similar to the so-called random waypoint (RWP) mobility model commonly used in studies of wireless ad hoc networks [JM96], [BW02], [BRS03], [NC04]. In the RWP model the nodes move along straight line segments from one waypoint to the next and the waypoints are assumed to be uniformly distributed in some convex domain. The similarity between the RWP process and the packet transfers with the shortest path routes is striking and we can utilise the readily available results from [HLV05] in this case. For curvilinear paths the situation, however, is more complicated and the new results derived in the present paper allow us to compute the resulting scalar packet flux (i.e., traffic load).

\section{Preliminaries}

In this section we introduce the necessary notation and definitions for analysing the transport of the packets and the resulting traffic load in the network. Let $\mathcal{A}$ denote the region where the network is located. The packet generation rate corresponding to traffic demands is defined as follows.

Definition 1 (traffic demands) The rate of flow of packets from a differential area element $d A$ about $\mathbf{r}_{1}$ to a differential area element $d A$ about $\mathbf{r}_{2}$ is $\lambda\left(\mathbf{r}_{1}, \mathbf{r}_{2}\right) \cdot d A^{2}$ ("traffic matrix").

Remark 2 The total packet generation rate is given by

$$
\Lambda=\int_{\mathcal{A}} d^{2} \mathbf{r}_{1} \int_{\mathcal{A}} d^{2} \mathbf{r}_{2} \lambda\left(\mathbf{r}_{1}, \mathbf{r}_{2}\right) .
$$

Each generated packet is transferred along a certain multihop path. More formally,

Definition 3 Set of paths denoted by $\mathcal{P}$ defines for all source destination pairs $\left(\mathbf{r}_{1}, \mathbf{r}_{2}\right)$ a unique path $p \in \mathcal{P}$. Furthermore, let $s\left(p, \mathbf{r}_{1}, \mathbf{r}_{2}\right)$ denote the distance from $\mathbf{r}_{1}$ to $\mathbf{r}_{2}$ along the path $p$.

Remark 4 The mean path length, i.e., the mean distance a packet travels, is given by

$$
\bar{\ell}=\frac{1}{\Lambda} \int_{\mathcal{A}} d^{2} \mathbf{r}_{1} \int_{\mathcal{A}} d^{2} \mathbf{r}_{2} \lambda\left(\mathbf{r}_{1}, \mathbf{r}_{2}\right) \cdot s\left(p, \mathbf{r}_{1}, \mathbf{r}_{2}\right)
$$

Example 5 For the shortest paths we have

$$
\bar{\ell}_{\mathrm{sp}}=\frac{1}{\Lambda} \int_{\mathcal{A}} d^{2} \mathbf{r}_{1} \int_{\mathcal{A}} d^{2} \mathbf{r}_{2} \lambda\left(\mathbf{r}_{1}, \mathbf{r}_{2}\right) \cdot\left\|\mathbf{r}_{2}-\mathbf{r}_{1}\right\| .
$$

Probably the most important quantity for our purposes is the packet arrival rate into the proximity of a given node. This is described by notion of scalar flux, which in turn is defined in terms of angular flux. These are similar to corresponding concepts of particle fluxes in physics, e.g., in neutron transport theory [BG70]. In our case, the packet fluxes depend on the traffic demands $\lambda\left(\mathbf{r}_{1}, \mathbf{r}_{2}\right)$ and the chosen paths $\mathcal{P}$, and are defined as follows:

Definition 6 Angular flux of packets at point $\mathbf{r}$ in direction $\theta$, denoted by $\Phi(\mathbf{r}, \theta)=\Phi(\mathcal{P}, \mathbf{r}, \theta)$, is equal to the rate at which packets flow in the angle interval $(\theta, \theta+d \theta)$ across a small line segment of the length $d s$ perpendicular to direction $\theta$ at point $\mathbf{r}$ divided by $d x \cdot d \theta$ in the limit $d x \rightarrow 0$ and $d \theta \rightarrow 0$. 


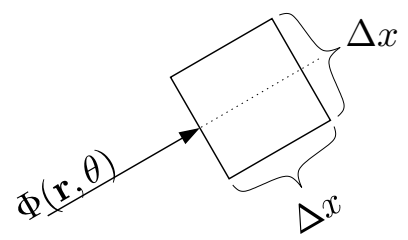

Fig. 1. Angular flux from direction $\theta$ multiplied by $\Delta x$ gives a specific packet arrival rate to a box with side length $\Delta x$.

Definition 7 Scalar flux of packets at point $\mathbf{r}$ is

$$
\Phi(\mathbf{r})=\Phi(\mathcal{P}, \mathbf{r})=\int_{0}^{2 \pi} \Phi(\mathcal{P}, \mathbf{r}, \theta) d \theta .
$$

Remark 8 Scalar flux of packets is equal to the rate at which packets enter a disk with diameter $d$ at point $\mathbf{r}$ divided by $d$ in the limit when $d \rightarrow 0$.

The proof follows trivially from the definitions. Also, in analogy with particle flux in physics the following holds.

Remark 9 For scalar flux $\Phi(\mathbf{r})$, packet density $n(\mathbf{r})$ and constant (local) velocity $v(\mathbf{r})$ it holds that

$$
\Phi(\mathbf{r})=n(\mathbf{r}) \cdot v(\mathbf{r}) .
$$

Proof: Packet arrival rate from a direction interval $(\theta, \theta+d \theta)$ across a line segment perpendicular to $\theta$ at $\mathbf{r}$ with length $\Delta x$ is equal to $\Phi(\mathbf{r}, \theta) \cdot \Delta x \cdot d \theta$ (cf., Fig. 1 and Def. 6). Each packet spends time $\Delta x / v(\mathbf{r})$ inside the box, and, according to Little's result, the mean number of packets in the box arriving from direction $(\theta, \theta+d \theta)$ is $\Phi(\mathbf{r}, \theta) \cdot \Delta x^{2} / v(\mathbf{r}) \cdot d \theta$ contributing an amount of $\Phi(\mathbf{r}, \theta) / v(\mathbf{r}) \cdot d \theta$ to the packet density. Integrating over $\theta$ gives $n(\mathbf{r})=\Phi(\mathbf{r}) / v(\mathbf{r})$.

Example 10 With uniform traffic demands and shortest path routing the resulting system is closely related to the random waypoint model (RWP). In the basic RWP model a node moves from one waypoint to another along the straight line segment and the waypoints are uniformly distributed in some convex domain with area $A$. The stationary node distribution is given by [HLV05]

$$
f_{\mathrm{RWP}}(\mathbf{r})=\frac{1}{2 \bar{\ell} A^{2}} \int_{0}^{2 \pi} d \theta a_{1} a_{2}\left(a_{1}+a_{2}\right),
$$

where $\bar{\ell}$ is the mean leg length, $a_{2}=a_{2}(\mathbf{r}, \theta)$ is the distance to the boundary in the direction $\theta$ and $a_{1}$ in the opposite direction (see Fig. 2). With $v=1$ the leg generation rate is equal to $1 / \bar{\ell}$, and hence the stationary node pdf of the RWP model is identical to the packet density $n(\mathbf{r})$ with an appropriate scaling,

$$
n(\mathbf{r})=\bar{\ell} \cdot f_{\mathrm{RWP}}(\mathbf{r}) \cdot \Lambda
$$

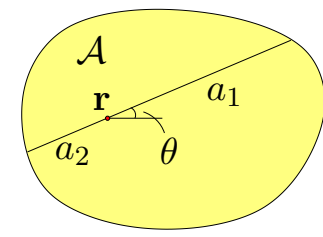

Fig. 2. Notation for node pdf (2) of the RWP model.

With $v=1$ we have $\Phi(\mathbf{r})=n(\mathbf{r})$ and the flux with uniform traffic demands and shortest paths is given by,

$$
\Phi(\mathbf{r})=\frac{\Lambda}{2 A^{2}} \int_{0}^{2 \pi} d \theta a_{1} a_{2}\left(a_{1}+a_{2}\right) .
$$

With this notation we can finally give a formal definition for the optimisation problem.

Definition 11 (load balancing problem) Find the set of paths, $\mathcal{P}_{\mathrm{opt}}$, minimising the maximum scalar flux,

$$
\mathcal{P}_{\text {opt }}=\underset{\mathcal{P}}{\arg \min } \max _{\mathbf{r}} \Phi(\mathcal{P}, \mathbf{r}) .
$$

Remark 12 (optimal maximum traffic load) With the load balanced paths the maximum load is

$$
\Phi_{\text {opt }}=\max _{\mathbf{r}} \Phi\left(\mathcal{P}_{\text {opt }}, \mathbf{r}\right)=\min _{\mathcal{P}} \max _{\mathbf{r}} \Phi(\mathcal{P}, \mathbf{r}) .
$$

In the above defintion, Def. 11, one needs the scalar flux $\Phi(\mathcal{P}, \mathbf{r})$. We will show in Section IV how this can be calculated for a given set of paths $\mathcal{P}$. Finding the optimal paths is a difficult problem of calculus of variation. In this paper, we do not search for a general solution but rather study three heuristically chosen families of paths and compare their performance with that of the shortest paths and with the bounds introduced in the next section.

\section{LOWER BOUNDS FOR PACKET FLUX}

Our next goal is to derive two lower bounds for achievable load balancing, i.e., for a given traffic pattern $\lambda\left(\mathbf{r}_{1}, \mathbf{r}_{2}\right)$ we want to find bounds for the minimum of the maximal traffic load that can be obtained by a proper choice of paths. Let us start by an illustrative example.

Example 13 Consider an $h \times w$ rectangle where packets are generated at rate $\Lambda$ at the bottom of the area (uniformly) as illustrated in Fig. 3. All packets travel directly up to the top of the area, i.e., we have shortest paths with mean path length of $\bar{\ell}=h$. The arrival rate of packets across any horizontal line segment of length $t$ is equal to $\Lambda \cdot t / w$. Hence, the flux at any point is constant, $\Phi=\Lambda / w=\Lambda \bar{\ell} / A$, and we have a perfect load balancing (cf. Def. 11). 


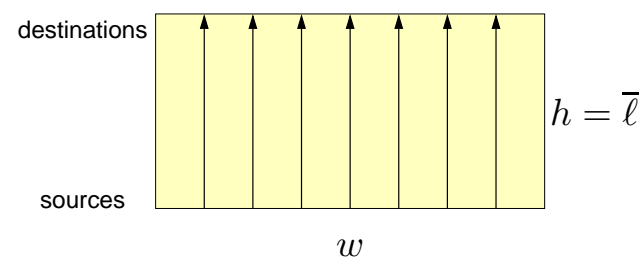

Fig. 3. Packets moving uniformly from the bottom to top of the area yield a uniform scalar flux of $\Phi=\Lambda / w=\Lambda \cdot \bar{\ell} / A$.

Note that the quantity $\Lambda \cdot \bar{\ell} \cdot \Delta t$ corresponds to the cumulative distance the packets arriving during a time interval of $\Delta t$ have to travel on average in order to reach their respective destinations. Consequently, the previous example suggests the following proposition.

\section{Proposition 14 (distance bound)}

$$
\max _{\mathbf{r}} \Phi(\mathcal{P}, \mathbf{r}) \geq \frac{\Lambda \cdot \bar{\ell}}{A}
$$

Proof: Without loss of generality we can set $v=1$ whence $\Phi(\mathbf{r})=n(\mathbf{r})$. Let $A$ be the area of $\mathcal{A}$ and $\bar{n}$ the mean density of packets, $\bar{n}=\bar{N} / A$, where $\bar{N}$ is the mean number of packets in the system. With $v=1$, Little's result implies $\bar{N}=\Lambda \cdot \bar{\ell}$. Hence

$$
\max _{\mathbf{r}} \Phi(\mathbf{r}) \geq \bar{n}=\frac{\bar{N}}{A}=\frac{\Lambda \cdot \bar{\ell}}{A} .
$$

Remark 15 For $\mathcal{P}_{\mathrm{sp}}$ consisting of straight line segments between the source destination pairs, we obtain the lowest possible value for $\bar{\ell}=\bar{\ell}_{\mathrm{sp}}$. Consequently,

$$
\Phi_{\mathrm{opt}} \geq \frac{\Lambda \cdot \bar{\ell}_{\mathrm{sp}}}{A}
$$

Alternatively, we can only consider traffic flows crossing an arbitrary boundary (cf., cut bound in wired networks).

Proposition 16 (cut bound) For any curve $\mathcal{C}$ which separates the domain $\mathcal{A}$ into two disjoint domains $\mathcal{A}_{1}$ and $\mathcal{A}_{2}$ it holds that

$$
\Phi_{\mathrm{opt}} \geq \frac{1}{L} \int_{\mathcal{A}_{1}} d^{2} \mathbf{r}_{1} \int_{\mathcal{A}_{2}} d^{2} \mathbf{r}_{2}\left(\lambda\left(\mathbf{r}_{1}, \mathbf{r}_{2}\right)+\lambda\left(\mathbf{r}_{2}, \mathbf{r}_{1}\right)\right),
$$

where $L$ is the length of the curve $\mathcal{C}$.

Proof: Consider first a short line segment $d s$ at $\mathbf{r}$ at some point along the curve $\mathcal{C}$. Let $\gamma$ denote a direction perpendicular to the curve at $\mathbf{r}$ such that the packets arriving from the angles $(\gamma-\pi / 2, \gamma+\pi / 2)$ cross $d s$ from outside to inside, and packets arriving from $(\gamma+$
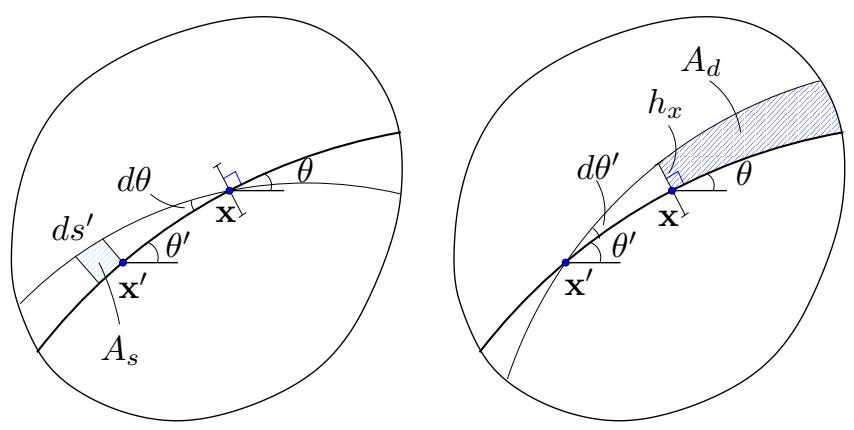

Fig. 4. Derivation of expression for the scalar flux.

$\pi / 2, \gamma+3 \pi / 2)$ cross $d s$ from inside to outside. The rate at which packets move across $d s$ is clearly given by

$$
\lambda(\mathbf{r}) d s=\int_{-\pi / 2}^{\pi / 2} \cos \alpha(\Phi(\mathbf{r}, \gamma+\alpha)+\Phi(\mathbf{r}, \gamma+\alpha+\pi)) d \alpha d s .
$$

As $0 \leq \cos \alpha \leq 1$ for $-\pi / 2 \leq \alpha \leq \pi / 2$ we get

$$
\begin{aligned}
\lambda(d s) & \leq \int_{-\pi / 2}^{\pi / 2} \Phi(\mathbf{r}, \gamma+\alpha)+\Phi(\mathbf{r}, \gamma+\alpha+\pi) d \alpha d s \\
& =\Phi(\mathbf{r}) d s \leq \max _{\mathbf{x} \in \mathcal{A}} \Phi(\mathbf{x}) d s .
\end{aligned}
$$

Integrating over the curve $\mathcal{C}$ completes the proof.

\section{PACKeT FluX With CURVILINEAR PATHS}

In this section, unless stated otherwise, we assume uniform traffic demands and a single path $p\left(\mathbf{r}_{1}, \mathbf{r}_{2}\right)$ between source and destination locations $\mathbf{r}_{1}$ and $\mathbf{r}_{2}$. Moreover, we assume the paths in $\mathcal{P}$ satisfy the so-called path continuity constraint:

\section{Definition 17 (path continuity)}

If $\mathbf{r} \in p\left(\mathbf{r}_{1}, \mathbf{r}_{2}\right)$, then $p\left(\mathbf{r}_{1}, \mathbf{r}_{2}\right)=p\left(\mathbf{r}_{1}, \mathbf{r}\right)+p\left(\mathbf{r}, \mathbf{r}_{2}\right)$.

The above definition lets us characterise the paths according to the direction at some point $\mathbf{x}$. In particular, the routing decision made in each point depends only on the destination of the packet, not the source. Let $p(\mathbf{x}, \theta)$ denote a path going through point $\mathbf{x}$ and having a direction $\theta$ at that point. The points along the curve (assumed to be smooth) are denoted by

$$
\mathbf{p}(\mathbf{x}, \theta, s), \quad \text { where } s \in\left[-a_{1}, a_{2}\right] \text {, and } a_{1}, a_{2}>0,
$$

so that $\mathbf{p}(\mathbf{x}, \theta, 0)=\mathbf{x}$. Thus, $a_{1}$ and $a_{2}$ denote the distance to the boundary along the path in opposite directions. Note that this means that we limit ourselves to paths that start and end at the boundary of the domain (no closed paths within the domain allowed). 
Definition 18 (path divergence) Let $h(\mathbf{x}, \theta, s)$ denote the rate with respect to the angle $\theta$ at which paths diverge at the distance of $s$,

$$
\begin{aligned}
h(\mathbf{x}, \theta, s) & =\lim _{d \theta \rightarrow 0} \frac{\|\mathbf{p}(\mathbf{x}, \theta+d \theta, s)-\mathbf{p}(\mathbf{x}, \theta, s)\|}{d \theta} \\
& =\left\|\frac{\partial}{\partial \theta} \mathbf{p}(\mathbf{x}, \theta, s)\right\| .
\end{aligned}
$$

Proposition 19 (angular flux with curvilinear paths) For a uniform traffic demands, $\lambda\left(\mathbf{r}_{1}, \mathbf{r}_{2}\right)=\Lambda / A^{2}$, the angular flux at point $\mathrm{x}$ in direction $\theta$ is given by

$$
\Phi(\mathbf{x}, \theta)=\frac{\Lambda}{A^{2}} \int_{0}^{a_{1}} \frac{h\left(\mathbf{x}, \theta,-s^{\prime}\right)}{h\left(\mathbf{x}^{\prime}, \theta^{\prime}, s^{\prime}\right)} \int_{0}^{a_{2}} h\left(\mathbf{x}^{\prime}, \theta^{\prime}, s+s^{\prime}\right) d s d s^{\prime} .
$$

Proof: Without loss of generality we may assume $\Lambda=1$. Our aim is to determine the angular flux in direction $\theta$ denoted by $\Phi(\mathbf{x}, \theta)$. To this end assume that a particular source is located in a differential area element about point $\mathbf{x}^{\prime}$ (see Fig. 4 left)

$$
\mathbf{x}^{\prime}=\mathbf{p}\left(\mathbf{x}, \theta, s^{\prime}\right), \quad s^{\prime} \leq 0,
$$

for which it clearly holds that

$$
\mathbf{p}\left(\mathbf{x}^{\prime}, \theta^{\prime}, s-s^{\prime}\right)=\mathbf{p}(\mathbf{x}, \theta, s) .
$$

Let $d \theta$ denote a differential angle at point $\mathbf{x}$ so that the differential source area about $x^{\prime}$ is given by (see Fig. 4 left)

$$
A_{s}=h\left(\mathbf{x}, \theta, s^{\prime}\right) \cdot d \theta \cdot d s^{\prime} .
$$

Similarly, let $d \theta^{\prime}$ denote a small angle at point $\mathbf{x}^{\prime}$, which yields a destination area of

$$
A_{d}=\int_{0}^{a_{2}} h\left(\mathbf{x}^{\prime}, \theta^{\prime}, s-s^{\prime}\right) d s d \theta^{\prime},
$$

as illustrated in Fig. 4 (right). The height of the "target line segment" perpendicular to the path at point $\mathrm{x}$ is

$$
h_{x}=h\left(\mathbf{x}^{\prime}, \theta^{\prime},-s^{\prime}\right) \cdot d \theta^{\prime} .
$$

Hence, the contribution to the angular flux from the differential source area is

$$
\begin{aligned}
d \Phi= & \frac{A_{s} \cdot A_{d}}{A^{2} \cdot d \theta \cdot h_{x}} \\
= & \frac{1}{A^{2}} \cdot \frac{1}{d \theta} \cdot\left(\frac{1}{h\left(\mathbf{x}^{\prime}, \theta^{\prime},-s^{\prime}\right) \cdot d \theta^{\prime}}\right) . \\
& \left(h\left(\mathbf{x}, \theta, s^{\prime}\right) \cdot d \theta \cdot d s^{\prime}\right) \cdot \int_{0}^{a_{2}} h\left(\mathbf{x}^{\prime}, \theta^{\prime}, s-s^{\prime}\right) d s d \theta^{\prime} \\
= & \frac{1}{A^{2}} \cdot \frac{h\left(\mathbf{x}, \theta, s^{\prime}\right)}{h\left(\mathbf{x}^{\prime}, \theta^{\prime},-s^{\prime}\right)} \cdot \int_{0}^{a_{2}} h\left(\mathbf{x}^{\prime}, \theta^{\prime}, s-s^{\prime}\right) d s d s^{\prime} .
\end{aligned}
$$

Hence, the angular flux at $\mathbf{x}$ in direction $\theta$ is given by

$\Phi(\mathbf{x}, \theta)=\frac{1}{A^{2}} \int_{-a_{1}}^{0} \frac{h\left(\mathbf{x}, \theta, s^{\prime}\right)}{h\left(\mathbf{x}_{1}, \theta^{\prime},-s^{\prime}\right)} \int_{0}^{a_{2}} h\left(\mathbf{x}^{\prime}, \theta^{\prime}, s-s^{\prime}\right) d s d s^{\prime}$.

The proposition follows upon substition $s^{\prime} \leftarrow-s^{\prime}$.

Remark 20 (angular flux with non-uniform $\lambda\left(\mathbf{r}_{1}, \mathbf{r}_{2}\right)$ ) It is straightforward to generalise (5) to the case of non-uniform traffic demands $\lambda\left(\mathbf{r}_{1}, \mathbf{r}_{2}\right)$. In this case, the angular flux at point $\mathrm{x}$ in direction $\theta$ is given by

$$
\begin{aligned}
\Phi(\mathbf{x}, \theta) & =\int_{0}^{a_{1}} \frac{h\left(\mathbf{x}, \theta,-s^{\prime}\right)}{h\left(\mathbf{x}^{\prime}, \theta^{\prime}, s^{\prime}\right)} . \\
& \int_{0}^{a_{2}} \lambda\left(\mathbf{x}^{\prime}, \mathbf{p}\left(\mathbf{x}^{\prime}, \theta^{\prime}, s+s^{\prime}\right)\right) \cdot h\left(\mathbf{x}^{\prime}, \theta^{\prime}, s+s^{\prime}\right) d s d s^{\prime} .
\end{aligned}
$$

Example 21 For the shortest paths we have

$$
h(\mathbf{x}, \theta, s)=\|s\|,
$$

and consequently, for uniform traffic demands, the angular flux is given by

$$
\begin{aligned}
\Phi(\mathbf{x}, \theta) & =\frac{\Lambda}{A^{2}} \int_{0}^{a_{1}} \int_{0}^{a_{2}}\left(s+s^{\prime}\right) d s d s^{\prime} \\
& =\frac{\Lambda}{A^{2}} \int_{0}^{a_{1}} a_{2}^{2} / 2+a_{2} s^{\prime} d s^{\prime}=\frac{\Lambda}{2 A^{2}} a_{1} a_{2}\left(a_{1}+a_{2}\right),
\end{aligned}
$$

in accordance with [HLV05] as presented in Ex. 10.

\section{EXAMPLE: UNIT DISK WITH UNIFORM DEMANDS}

In this section we will demonstrate how the proposed framework can be applied. To this end, we consider a special case of a unit disk with uniform load,

$$
\mathcal{A}=\left\{\mathbf{r} \in \mathbb{R}^{2}:|\mathbf{r}|<1\right\}, \text { and, } \lambda\left(\mathbf{r}_{1}, \mathbf{r}_{2}\right)=\Lambda / \pi^{2} .
$$

We study the performance of three simple families of paths: outer and inner radial ring paths and circular paths. The performance of these path sets is compared with that of the shortest paths, and with the appropriate lower bounds for the minimal maximum traffic load.

Example 22 (unit disk with shortest paths) For transport according to the straight line segments we can rely on the results for the RWP model (see Ex. 10 and [HV05]). At distance d, the scalar flux is given by

$$
\Phi_{\mathrm{sp}}(d)=\frac{2\left(1-d^{2}\right) \cdot \Lambda}{\pi^{2}} \int_{0}^{\pi} \sqrt{1-d^{2} \cos ^{2} \phi} d \phi .
$$


In particular, the maximum flux is obtained at the center,

$$
\Phi_{\mathrm{sp}}(0)=\frac{2}{\pi} \cdot \Lambda \approx \underline{0.6366 \cdot \Lambda} .
$$

Example 23 (distance bound for unit disk) The distance bound gives us a relationship between the obtainable maximum load and the mean path length. With shortest paths we have $\bar{\ell}_{\mathrm{sp}}=128 / 45 \pi$ which upon substitution in (4) yields

$$
\Phi_{\mathrm{opt}} \geq \frac{\Lambda \cdot 128}{45 \pi^{2}} \approx \underline{0.2882 \cdot \Lambda} .
$$

\section{Example 24 (upper bound for mean path length)}

The known traffic load when shortest paths are used can be combined with the distance bound. According to (6)

$$
\Phi_{\mathrm{sp}}=\frac{2 \Lambda}{\pi}=\frac{\Lambda \cdot 2}{A} .
$$

On the other hand, according to (3) we have

$$
\max _{\mathbf{r}} \Phi(\mathcal{P}, \mathbf{r}) \geq \frac{\Lambda \cdot \bar{\ell}}{A}=\Phi_{\mathrm{sp}} \cdot \frac{\bar{\ell}}{2} .
$$

Hence, if with the given paths $\mathcal{P}$ the mean path length $\bar{\ell}>2$, then the resulting maximum traffic load is greater than the one obtained with the shortest paths.

Example 25 (cut bound) Let the separating curve $\mathcal{C}$ be a concentric circle with radius $d, 0<d<1$. For the packet rate across the boundary it holds that

$$
\lambda(d) \geq 2 d^{2}\left(1-d^{2}\right) \cdot \Lambda,
$$

which corresponds to radial flux (per unit length)

$$
\Phi_{r}(d)=\frac{2 d^{2}\left(1-d^{2}\right)}{2 \pi d} \cdot \Lambda=\frac{d-d^{3}}{\pi} \cdot \Lambda \leq \Phi_{\mathrm{opt}} .
$$

As this is a lower bound for the scalar flux we want to maximise it. The tightest lower bound is obtained with $d=1 / \sqrt{3}$,

$$
\Phi_{\mathrm{opt}} \geq \frac{2}{3 \sqrt{3} \cdot \pi} \cdot \Lambda \approx \underline{0.1225 \cdot \Lambda} .
$$

Hence, in this case, if comparing to the shortest paths, the cut bound gives a lower bound which says that no more than $80 \%$ improvement in the maximum load is possible, while according to the distance bound at most a $55 \%$ improvement is possible.

\section{A. Radial Ring Paths}

Let us next consider three actual path sets as illustrated in Fig. 5. The shortest paths (SP) are equivalent to RWP model as has been already mentioned. The two radial path sets are similar in that each path consists of two sections. One section is a radial path towards (or away from) the origin, and the other section is an angular path along a ring with a given radius. The difference between the two sets is in the order of components, "Rout" uses the outer angular rings and "Rin" the inner ones, as the names suggest. Note that locally, at any point, the packets are transmitted only in 4 possible directions (2 radial and 2 angular), which may simplify the possible implementation of the time division multiplexing.

When studying the arrival rate into a small area at the distance of $d$ from the origin one needs to consider both radial and angular ring movement. The radial component of the flux is the same for both path sets, i.e.,

$$
\Phi_{r}(d)=\frac{d-d^{3}}{\pi} \cdot \Lambda
$$

1) Outer radial ring paths: Let us next consider outer radial ring paths. We want to determine the flux along the ring at the distance of $d$. To this end, consider a small line segment from $(-d, 0)$ to $(-d-\Delta, 0)$ as "target area" as illustrated in Fig. 6. Packets originating from a small "source" area at the distance of $d$ in direction $\theta$ travel through the target line segment if their destination is in "destination" area. The size of the source area is

$$
d \cdot \Delta \cdot d \theta
$$

while the possible destination area is

$$
\frac{\theta \cdot \pi d^{2}}{2 \pi}=\frac{d^{2}}{2} \cdot \theta
$$

Combining these with (7) yields the angular flux at the distance of $d$,

$$
\Phi_{\theta}(d)=\frac{4 \Lambda}{\Delta \pi^{2}} \int_{0}^{\pi} \frac{d^{2}}{2} \theta \Delta d \theta=d^{3} \cdot \Lambda .
$$

Hence, the total flux at the distance of $d$ for outer path set is given by

$$
\Phi_{\text {Rout }}(d)=\Phi_{r}(d)+\Phi_{\theta}(d)=\frac{(\pi-1) d^{3}+d}{\pi} \cdot \Lambda .
$$

The maximum flux is obtained at point $d=1$,

$$
\Phi_{\text {Rout }}(1)=\Lambda \text {. }
$$



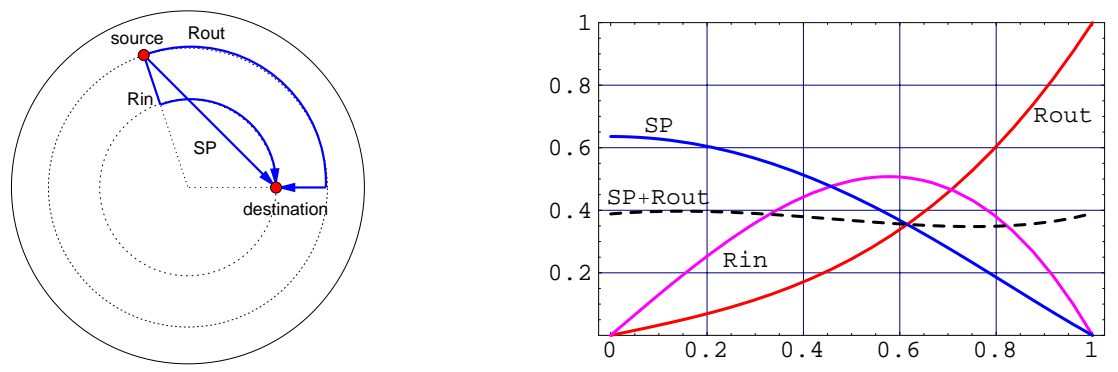

Fig. 5. Left figure illustrates the three path sets considered: straight line segments (SP), radial paths with outer (Rout) and inner (Rin) angular ring transitions. In right figure the resulting flux is plotted for for the three path sets (SP, Rout and Rin) and for a randomised combination of SP and Rout (dashed curve) as a function of distance $d$ from the center.

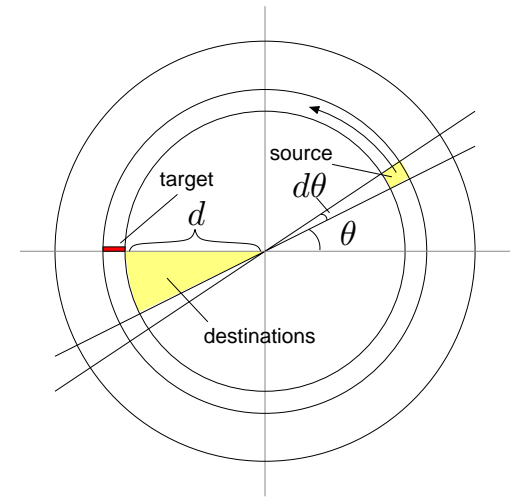

Fig. 6. Derivation of the angular ring flux at the distance of $d$ for outer radial ring paths.

2) Inner radial ring paths: For inner paths the possible destination area of packets is

$$
\frac{1-d^{2}}{2} \cdot \theta
$$

and

$$
\begin{aligned}
\Phi_{\theta}(d) & =\frac{4 \Lambda}{\Delta \pi^{2}} \int_{0}^{\pi} \frac{1-d^{2}}{2} \theta \cdot d \Delta d \theta \\
& =\frac{2\left(d-d^{3}\right) \cdot \Lambda}{\pi^{2}} \int_{0}^{\pi} \theta d \theta=\left(d-d^{3}\right) \cdot \Lambda .
\end{aligned}
$$

Consequently, combining the above with (7) gives

$$
\Phi_{\operatorname{Rin}}(d)=(1+1 / \pi)\left(d-d^{3}\right) \cdot \Lambda .
$$

The maximum is obtained at point $d=1 / \sqrt{3}$,

$$
\Phi_{\operatorname{Rin}}(1 / \sqrt{3}) \approx \underline{0.507 \cdot \Lambda} .
$$

Hence, the outer version leads to a higher maximum load than the shortest paths while the inner version yields a slightly better solution. The resulting packet fluxes are illustrated in Fig. 5 (right) as a function of the distance $d$ from the center.
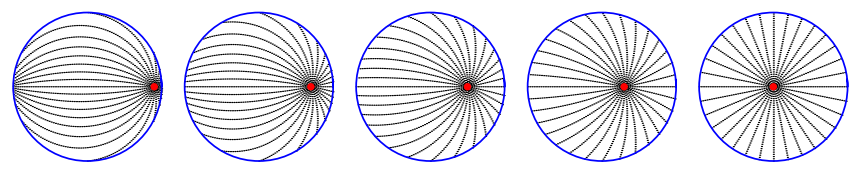

Fig. 8. Circular paths illustrated for different destination (or source) points.

\section{B. Circular paths}

As the last path set we consider curvilinear paths, referred to as circular paths, which consist of such sections of circumference of circles that cross the unit disk at the opposite points as illustrated in Fig. 7. Some example paths are illustrated in Fig. 8 for different destination (or source) locations. From the figure it can be seen that these paths smoothly move some portion of the traffic away from the center of the area.

The angular flux can be calculated using Proposition 19 , and the scalar flux is obtained by integration (cd. Definition 7). The resulting scalar flux is depicted in Fig. 7 (right). It can be seen that the traffic load is fairly well distributed in the area. The maximum flux is obtained at the center of the disk, where the flux is 0.4244. In fact, it is possible to determine the packet flux at the center analytically. For this we have

$$
\Phi(0)=\frac{4}{3 \pi} \cdot \Lambda, \quad(\approx \underline{0.4244 \cdot \Lambda})
$$

which is exactly $2 / 3$ of the flux with the shortest paths consisting of straight line segments (cf. Ex. 22).

\section{Randomised path selection approach}

One option to achieve a lower maximum load is to allow the use of several paths for each pair of nodes (similarly as in [PP03], [GK04]). In particular, let us relax our assumptions a bit and assume a finite number of path sets $\left\{\mathcal{P}_{i}\right\}$, where $i=1, \ldots, n$. Upon transmission of a packet the source node chooses a path from path set $i$ with probability of $i, i=1, \ldots, n$. 

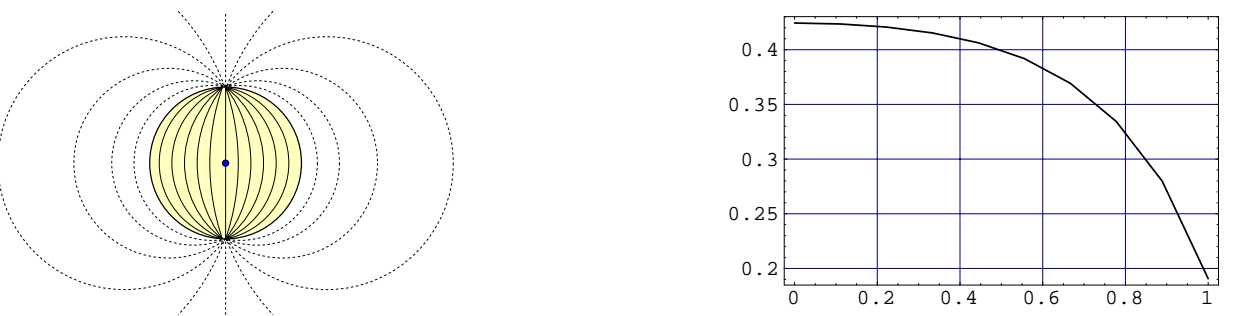

Fig. 7. Circular paths are paths formed by the circumferences of circles which cross the unit disk at the opposite points. The figure on right illustrates the resulting flux as a function of distance from the center.

Proposition 26 (packet flux with randomised paths) $A$ randomised path selection from path sets $\left\{\mathcal{P}_{i}\right\}$, $i=1, \ldots, n$, upon transmission with probabilities $p_{i}$, $i=1, \ldots, n$ yields a total scalar flux of

$$
\Phi(\mathbf{r})=\sum_{i} p_{i} \cdot \Phi\left(\mathcal{P}_{i}, \mathbf{r}\right)
$$

Example 27 Consider uniform traffic demands in unit disk and two path sets, 1) shortest paths, and 2) the outer radial paths. Weights $p_{1}=0.6$ and $p_{2}=0.4$ give a packet flux of

$$
\Phi(d)=0.61 \cdot \Phi_{\mathrm{sp}}(d)+0.39 \cdot \Phi_{\text {Rout }}(d) .
$$

The resulting flux is almost constant as illustrated by the dashed line in Fig. 5. The maximum is $0.397 \cdot \Lambda$, which is about $37 \%$ lower than with the shortest paths.

Example 28 The same technique can be taken further, e.g., by combining all three path sets as follows

$\Phi(d)=0.52 \cdot \Phi_{\mathrm{sp}}(d)+0.37 \cdot \Phi_{\mathrm{Rout}}(d)+0.11 \cdot \Phi_{\operatorname{Rin}}(d)$, which gives a maximum flux of $0.379 \cdot \Lambda$, i.e., about $40 \%$ lowering in the maximum flux when compared to the shortest paths.

\section{CONCLUSIONS}

In this paper we have presented a general framework for analysing traffic load and routing in a large dense multihop network. The approach relies on strong separation of spatial scales between the microscopic level, corresponding to the node and its immediate neighbours, and the macroscopic level, corresponding to the path from the source to the destination. In a dense wireless network with this property the local traffic load can be assimilated with the so-called scalar packet flux. The packet flux is bounded by a maximal value that the network with a given MAC and packet forwarding protocol can sustain. The packet flux depends on traffic pattern $\lambda\left(\mathbf{r}_{1}, \mathbf{r}_{2}\right)$ and the chosen set of routing paths $\mathcal{P}$. The load balancing problem thus comprises of determining the set of routing paths such that the maximal value of the flux in the network is minimised. While the general solution of this difficult problem remains for future work, our main contribution in this paper consists of giving bounds for the packet flux and giving a general expression for determining the packet flux at a given point when a given set of curvilinear paths is used. The results are illustrated by numerical examples with three different sets of paths in unit disk. Future work includes investigating how to find nearly optimal load balancing in a distributed fashion.

\section{REFERENCES}

[BG70] G.I. Bell and S. Glasstone. Nuclear Reactor Theory. Reinhold, 1970.

[BRS03] C. Bettstetter, G. Resta, and P. Santi. The node distribution of the random waypoint mobility model for wireless ad hoc networks. IEEE Transactions on Mobile Computing, 2(3):257-269, July-September 2003.

[BW02] C. Bettstetter and C. Wagner. The spatial node distribution of the random waypoint mobility model. In Proc. of German Workshop on Mobile Ad Hoc networks (WMAN), Ulm, Germany, March 2002.

[DBT05] Olivier Dousse, François Baccelli, and Patrick Thiran. Impact of interferences on connectivity in ad hoc networks. IEEE/ACM Trans. Networking, 13(2):425-436, April 2005.

[GK04] Yashar Ganjali and Abtin Keshavarzian. Load balancing in ad hoc networks: single-path routing vs. multi-path routing. In Proc. of Infocom'04, Hong Kong, March 2004.

[HLV05] Esa Hyytiä, Pasi Lassila, and Jorma Virtamo. Spatial node distribution of the random waypoint mobility model with applications. IEEE Transactions on Mobile Computing. to appear in.

[HV05] Esa Hyytiä and Jorma Virtamo. Random waypoint mobility model in cellular networks. Springer Wireless Networks, 2005. to appear in.

[JM96] David B. Johnson and David A. Maltz. Dynamic source routing in ad hoc wireless networks. In Tomasz Imielinski and Hank Korth, editors, Mobile Computing, volume 353, pages 153-181. Kluwer Academic Publishers, 1996.

[NC04] W. Navidi and T. Camp. Stationary distributions for the random waypoint mobility model. IEEE Transactions on Mobile Computing, 3(1):99-108, January-March 2004.

[PP03] Peter P. Pham and Sylvie Perreau. Performance analysis of reactive shortest path and multi-path routing mechanism with load balance. In Proc. of Infocom'03, volume 1, pages 251-259, San Francisco, USA, March-April 2003.

[SMS05] Birsen Sirkeci-Mergen and Anna Scaglione. A continuum approach to dense wireless networks with cooperation. In Proc. of Infocom'05, Miami, FL, USA, 2005. 